Check for updates

Cite this: RSC Adv., 2017, 7, 45513

\title{
Merging orthogonal microfluidic flows to generate multi-profile concentration gradients $\uparrow$
}

\begin{abstract}
A. Menachery, (ID $\ddagger^{a}$ N. Kumawat (ID $\ddagger^{a}$ and M. A. Qasaimeh (iD *ab
This work describes a novel microfluidic device capable of generating multi-profile gradients that include sigmoidal, parabolic, and exponential concentration variations across its main channel. The main distinguishing feature of this device is its simple geometry: it contains fewer fluidic channels that provide versatility and ease of operation. The narrow orthogonal side channels transport analyte into a wider buffer stream, and by merely altering flow rates of either one or both streams, gradient profiles are switched from one to another. Finite element simulations match well with the experimental results and demonstrate simple manipulation of the generated gradients. Results show that the gradient's slope, extent, and position can be modulated by subtle flow rate variations, making the platform adaptable for various biological applications. The simplicity of the device offers potential for stable chemotactic studies for long durations.
\end{abstract}

Received 31st August 2017

Accepted 18th September 2017

DOI: 10.1039/c7ra09692e

rsc.li/rsc-advances

understanding of several chemotaxis-related biological processes, a gap still remains on explaining how precisely different biomolecular gradients influence cell responses and sensitivities during chemotaxis, and how multiple concentration gradients of different chemokines cooperate in guiding cells. Therefore, new systems that better mimic the in vivo microenvironment in a controlled and stable way, which allow for quantitative studies of gradient signaling, are in demand.

Various traditional methods have been previously employed to generate chemotactic gradients to characterize cell migration. Boyden proposed the transwell assay to understand the migration of leukocytes. ${ }^{11,12}$ This assay contains two chambers separated by a porous membrane through which cells migrate. Cells placed in the top chamber can migrate through the pores of the membrane into the lower chamber towards an attractant. In a Dunn chamber, two compartments are connected by a bridge, wherein, one chamber is loaded with cells resuspended in medium, while the other is filled with medium containing a chemoattractant. ${ }^{11,12}$ Although such traditional methods were very useful in shaping our current knowledge of gradient signaling in several biological processes, they lacked repeatability and precise control to generate quantifiable userdefined gradients.

Microfluidics has emerged as an alternative to overcome the drawbacks associated with traditional methods. Using various microfabrication technologies, well defined enclosed channels can be created by which various concentration profiles can be obtained both in static and dynamic "flow" modes. Microfluidics is ideally suited for use in the latter mode, where laminar fluid streams interact with each other at the interface by means of diffusion, providing stationary concentration profiles or well defined temporal changes. While it is also

\footnotetext{
Division of Engineering, New York University Abu Dhabi, P.O. Box 129188, Abu Dhabi, United Arab Emirates. E-mail: mohammad.qasaimeh@nyu.edu

${ }^{b}$ Department of Mechanical and Aerospace Engineering, Tandon School of Engineering, New York University, NY 11201, USA

$\dagger$ Electronic supplementary information (ESI) available. See DOI: 10.1039/c7ra09692e

\$ Joint first authors.
} 
possible to use the static flow mode for generating concentration gradients, the main associated drawback is the limited duration of gradient stability as diffusion flattens the gradients.

To date, several microfluidic platforms with different channel network architectures have been employed to generate linear and non-linear gradients. Examples include a device for generating static linear gradients which was developed by sandwiching a three-channel laser-cut membrane between a plastic reagent manifold and a glass substrate. ${ }^{13}$ In this device, fluid perturbations were minimized so that bacterial chemotaxis could be clearly studied. Designs that have been commonly reported for dynamic gradients include the Christmas tree-like microfluidic network, in which, joining and splitting of laminar fluid streams can yield linear and nonlinear profiles in the recombined main channel. ${ }^{\mathbf{1 4 1 5}}$ In an attempt to provide a more compact device layout, Yang et al. designed and tested a high-density network of radial channels to produce a stable and reproducible gradient with potential for high-throughput drug screening. ${ }^{16}$ We previously developed a floating concentration gradient using the microfluidic probe technology, where concentration gradients were generated in an open channel-less microfluidic configuration. ${ }^{17-19}$ Furthermore, to combine concentration gradients and isolation of the solute within small chambers, a ladder-shaped channel network with $2 \mathrm{~nL}$ small side chambers was developed. ${ }^{20}$ Using the aforementioned techniques among others, microfluidic gradient chips have the potential to further contribute to the understanding of immune cell migration, ${ }^{21}$ axonal navigation, ${ }^{22,23}$ stem cell differentiation, ${ }^{24}$ and bacterial chemotaxis. ${ }^{\mathbf{1 0 , 2 5}}$

In this work, as depicted in Fig. 1, we developed a microfluidic device that has a simple design capable of generating multi-profile gradients, such as sigmoid, parabolic and exponential profiles. This can be achieved by only altering fluid flow rates without the need for design modifications. The proposed design is a simple network of merged perpendicular channels that reduce complexity compared to previously reported designs. Therefore, the developed platform is easy to fabricate and operate with potential to scale up. The device consists of one main inlet and outlet, with two orthogonal side inlets that are further branched into four channels. Each of the four side channels has a non-linearly increasing length to alter the hydrodynamic resistance such that decreasing flow rates are generated in the main channel.
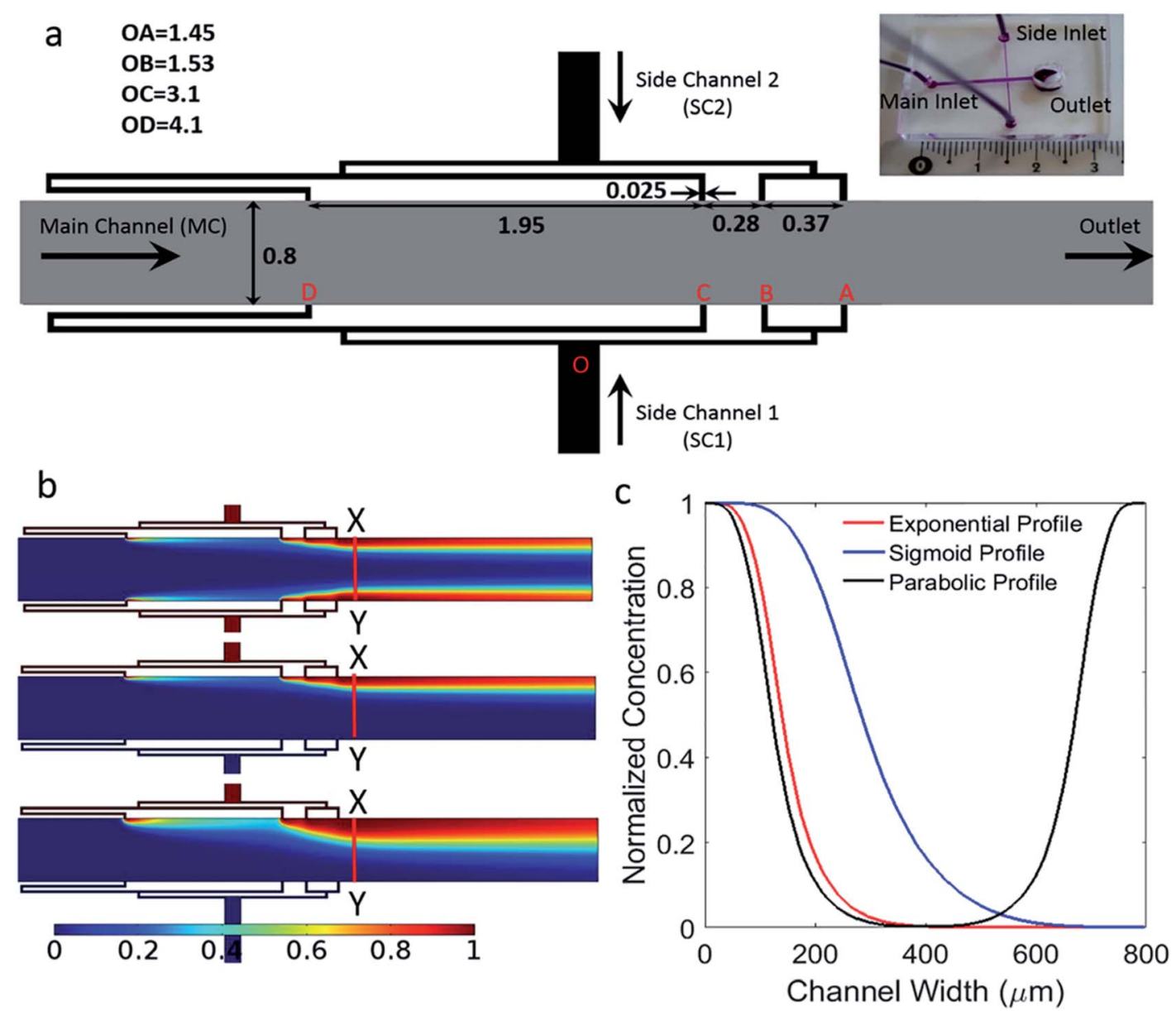

Fig. 1 The developed microfluidic device for generating multi-profile concentration gradients. (a) Schematic of the device (not to scale) featuring its components and dimensions $(\mathrm{mm})$ : one main inlet, two side inlets each branched into four orthogonal side channels, and one outlet. Inset shows device photograph containing dye within the channel structure. (b) COMSOL generated surface concentration profiles to produce parabolic, exponential and sigmoidal gradients (top-to-bottom). (c) Concentration profiles along XY lines in (b). 


\section{Materials and methods}

\section{Design}

The geometry of the device consists of a main flow channel with a width of $800 \mu \mathrm{m}$ and perpendicular side channels merging into the main channel, as shown in Fig. 1a. The depth of the channels is $40 \mu \mathrm{m}$. There are two main side channels, where each of them branch out into four. The width of each of these narrow branches is $25 \mu \mathrm{m}$. These side structures are identical and mirrored around the main channel. The four branches are represented as $\mathrm{OA}, \mathrm{OB}, \mathrm{OC}$ and $\mathrm{OD}$ and their lengths are 1.45, $1.53,3.1$ and $4.1 \mathrm{~mm}$, respectively. The side channel ' $\mathrm{A}$ ' which is closest to the outlet, is placed at a distance of $3.2 \mathrm{~mm}$. This distance gives sufficient length to perform future chemotaxis studies. The distances AB, BC and CD are 0.37, 0.28 and 1.95 $\mathrm{mm}$, respectively.

\section{Microfabrication}

The SU-8 mold was fabricated using SU-8 2025 (MicroChem USA) on a 4 -inch silicon wafer. The first step of the process was spin-coating the photo resist to a thickness of $40 \mu \mathrm{m}$ with a spin speed of $3000 \mathrm{rpm}$ for $30 \mathrm{~s}$. The deposited layer was then soft baked at $65{ }^{\circ} \mathrm{C}$ for 2 minutes and at $95{ }^{\circ} \mathrm{C}$ for 5 minutes. The resist was exposed to a UV dose of $150 \mathrm{~mJ} \mathrm{~cm}^{-2}$ at $365 \mathrm{~nm}$, after which, a post-exposure bake was performed for 2 minutes at $65{ }^{\circ} \mathrm{C}$ and 5 minutes at $95{ }^{\circ} \mathrm{C}$. The unexposed SU-8 photo resist was developed using a standard SU-8 developer, following which the structure was rinsed with isopropanol. In the final step, a hard bake was performed at $150{ }^{\circ} \mathrm{C}$ to remove any surface cracks by means of annealing. Subsequently, degassed polydimethylsiloxane (PDMS) (Sylgard 184), in a 10:1 ratio of prepolymer to curing agent was poured over the SU-8 mold. The PDMS was then baked at $75{ }^{\circ} \mathrm{C}$ for 1 hour and allowed to cool down to room temperature before it was peeled off and bonded to a glass substrate by means of an oxygen plasma.

\section{D finite element analysis}

The simulations were carried out using a commercially available finite element simulation software COMSOL Multiphysics v4.3 (COMSOL, USA). The two physics modules used were 'Laminar Flow' and 'Transport of Diluted Species', and the device was modeled with the same dimensions as the microfabricated channel structure. The material properties, such as dynamic viscosity and density, were defined using the characteristics of water. The diffusion coefficient of the solute is $5.4 \times$ $10^{-6} \mathrm{~cm}^{2} \mathrm{~s}^{-1}$, which corresponds to the diffusion constant of fluorescein sodium salt in water. ${ }^{26}$ The results were obtained under steady-state conditions. For the flow module, we defined no-slip boundary conditions on the glass substrate and the sides of the channel. The flow rates used were matched to the experimental settings using a zero-pressure boundary condition at the outlet. The boundary conditions for solute concentrations were defined as 1 for the fluorescein source and zero for the water source to obtain concentration gradients between these values.

\section{Experimental procedures and analysis}

The fluorescein solution was prepared by dissolving fluorescein sodium salt in deionized (DI) water to prepare a final concentration of $1 \mathrm{mg} \mathrm{mL}{ }^{-1}$. The fluorescein solution and DI water were introduced into the main and side inlets using PTFE tubes connected to a syringe pump (NEMESYS, Cetoni Gmbh, Germany). The experiments were performed on a Nikon SMZ18 fluorescent stereo microscope (SM). Color images were obtained using microscope's CCD camera and analyzed with a custom code written in MATLAB (MathWorks, USA). Intensity values were obtained along a line cross-section determined by the user. Results were subsequently normalized between 0 and 1 where the pixel intensity for zero was obtained from the main channel prior to mixing and the intensity value for 1 was obtained from the side channel that is supplying the fluorescein solution.

\section{Results and discussion}

\section{Device design and optimization}

The channel structure of the device was conceived with the intention of developing a simple fluidic device without several branching channels employed in tree geometries and ladder networks. Orthogonal channels within a laminar flow device will result in the merging of fluid streams with well-defined flow profiles that are advection and diffusion limited. The developed device consists of one main inlet and one outlet, with two orthogonal side inlets that are further divided into four. This way, parabolic concentration gradient profile can be achieved by activating both side channels to flow solutions of chemokines. When generating sigmoid or exponential concentration gradient profiles, only one side channel is activated and used for flowing a chemokine solution, while the other side channel is kept inactivated. In all cases, the main channel inlet is used for flowing buffer.

The use of narrow side channels instead of wide channels enables a smooth transition of the fluid into the main channel without any recirculation zones and backflow problems. A wide main channel was used so that future biological studies could be performed on larger areas where cells can migrate over long distances defined by the length of the gradient across the main channel. Branching each of the side channels into four enabled the production of a smooth concentration gradient. Fig. 2a and $\mathrm{b}$ demonstrate that, unlike the abrupt change in gradient obtained when using single side channel, the current design results in a gradual and smooth increase in the concentration gradient as the number of channels is increased. This is more representative of commonly used exponential functions.

Before attaining the current geometry, optimization was carried out primarily for the lengths of the side channels and their separation distance along the main channel. The fluid output from the side branches is a function of two dependent variables, the lengths of the branches and their separation distance along the main channel. It is important to note that variation of flow rates from individual branches can be achieved by either varying their lengths, varying their separation distance 


\section{a}

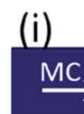

$\underbrace{}_{2} \times 1$ Side Channel

$$
\text { SC2 } \downarrow
$$

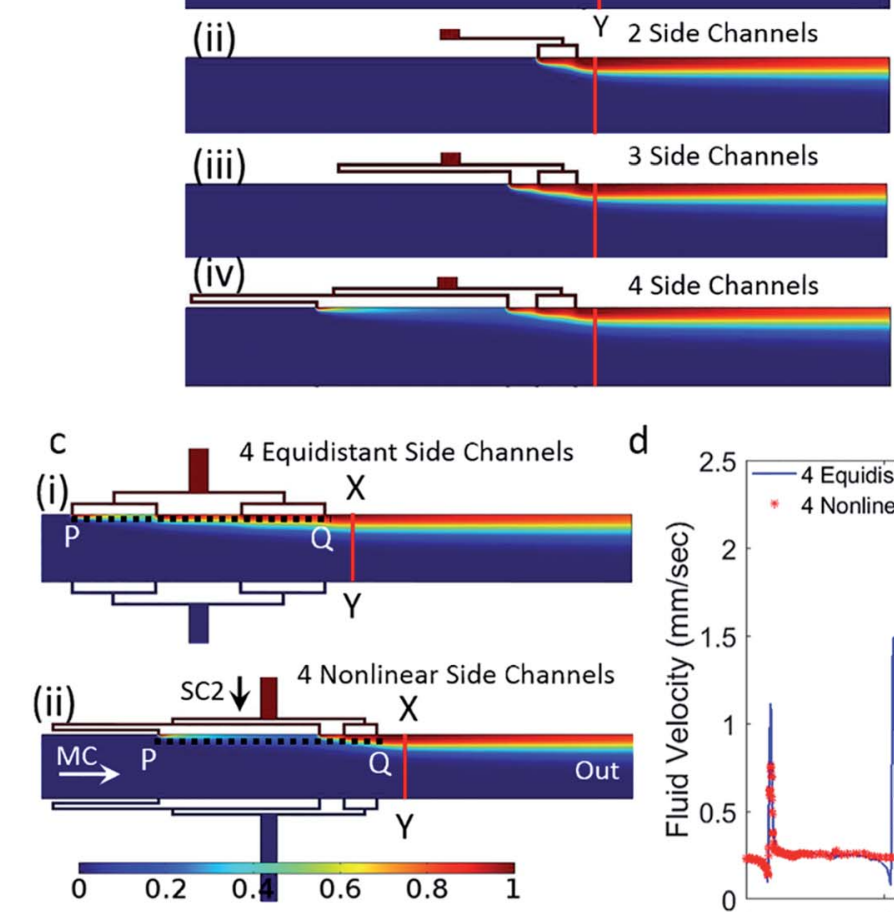

政 d

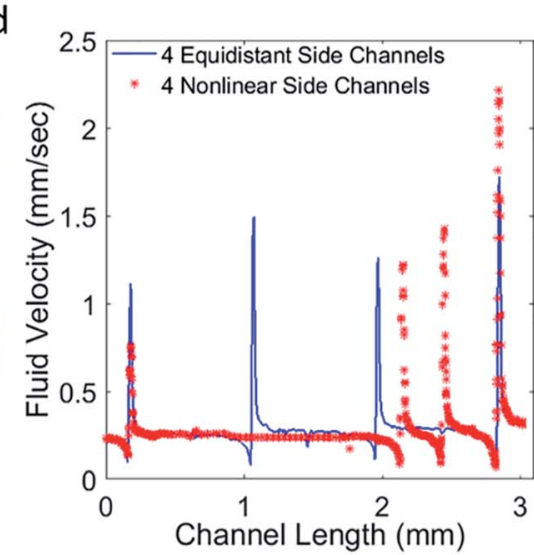

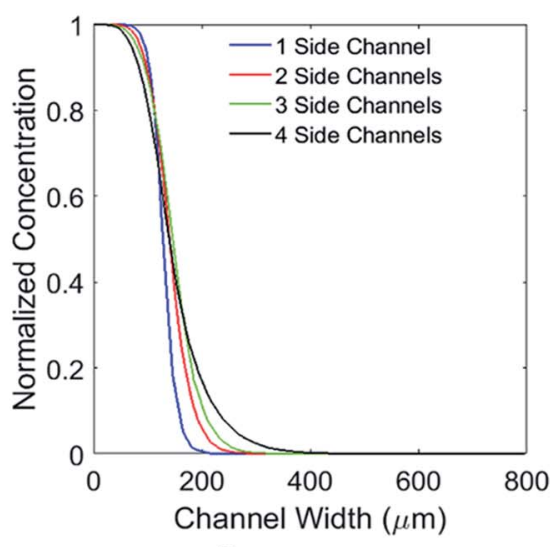

e

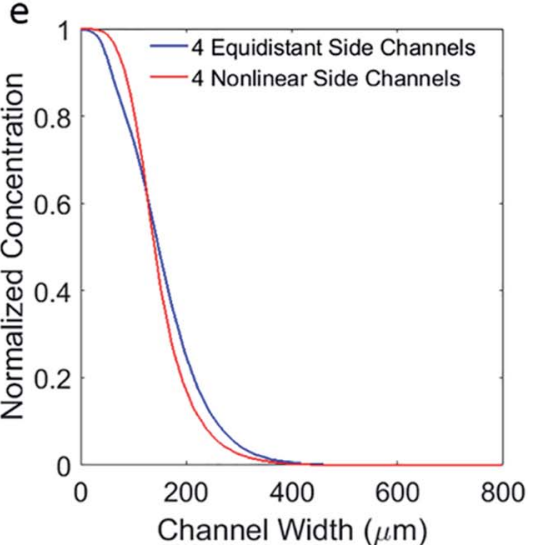

Fig. 2 Optimizing branches of the orthogonal side channels. (a) COMSOL generated exponential concentration profiles for a single side channel and 2, 3 and 4 branches. (b) Concentration profiles along corresponding $X Y$ lines in (a). (c) Simulated surface concentration plots for four equidistant branches with equal spacing and lengths and nonlinear branches with unequal spacing and lengths. (d) Velocity profiles along the dotted $P Q$ line shown in (c). (e) Exponential concentration gradient profiles plotted along $X Y$ lines shown in (c).

along the main channel, or a combination of the two. The length of the branches $\mathrm{OA}, \mathrm{OB}, \mathrm{OC}$ and $\mathrm{OD}$, and their spacing $\mathrm{AB}, \mathrm{BC}$ and $\mathrm{CD}$ (Fig. 1a) were chosen to achieve increasing flow rates from the branches downstream the channel (i.e. lowest flow rate from port $\mathrm{D}$ and highest flow rate from port $\mathrm{A}$ ). Increasing flow rates correspond to smooth variations in concentration along the length of the main channel.

To illustrate the difference between the proposed nonlinear and equidistant branching of the side channels, Fig. 2c, we constructed both models in COMSOL and calculated the fluid flow velocity across the branches (Fig. 2d), and evaluated the obtained concentration gradients in the main channel (Fig. 2e). The length from the first branched side inlet (A) to the fourth branched side inlet (D), is kept identical for both configurations highlighted as the length PQ in Fig. 2c. Here, it is evident that increasing velocity profiles cannot be obtained from an equidistant channel spacing. For non-linearly spaced channels, the velocity profile was found to be stable in the main channel immediately after the side channels and all the way to the outlet port. The corresponding concentration profiles for both configurations are shown in Fig. 2e. The line plot corresponding to the equidistant configuration exhibits a sudden change of slope in the upper region of the curve, which is not seen for the current geometry. This indicates that increasing velocity profiles correspond to smooth variations in the generated concentration gradient. Advection based chaotic mixing does not take place in the laminar flow system as shown by the stream lines in Fig. S1. $\dagger$ Mass transport in the lateral direction of the flow is dominated by diffusion occurring across the laminar streams introduced by the side and main channels. The flow rates from the side channels A, B, C, D increases as it moves away from the inlet such that $Q_{\mathrm{A}}>Q_{\mathrm{B}}>Q_{\mathrm{C}}>Q_{\mathrm{D}}$. As a result of this increasing flow rate, the gradient slope changes gradually along the various non-linear gradient profiles demonstrated in this work.

It is important that laminar flow devices provide cells with physiologically suitable shear stress environments. In a typical microfluidic device, cell may experience fluid shear stresses of the order of 0.001-10 dynes per $\mathrm{cm}^{2}$ for extended durations. ${ }^{27}$ The maximum fluid shear stress in a microfluidic channel, ${ }^{27}$ which occurs at the walls, can be determined using:

$$
\tau_{\max }=\frac{6 \mu Q}{w h^{2}},
$$

where $\tau_{\max }$ is shear stress induced by the flow, $\mu$ is the dynamic viscosity of water at $25{ }^{\circ} \mathrm{C}$ and $Q$ is the average flow rate within a channel of height $h$ and width $w$.

In the experiments reported here, Reynolds numbers in the range of 0.006 to 0.04 were used for creating exponential 
gradients, and the calculated corresponding shear stresses were within the range 0.1-0.8 dynes per $\mathrm{cm}^{2}$.

Advection is responsible for spatially regulating the effect of diffusion, thereby altering concentration profiles at various flow rates. For example, upon simulation of a concentration profile generated with a main channel flow of $480 \mu \mathrm{L} \mathrm{h}^{-1}$ and a side channel of $120 \mu \mathrm{L} \mathrm{h}^{-1}$, as shown in Fig. S2, † a large dye saturation region was created along with curves containing steep slopes compared with a main channel flow of $48 \mu \mathrm{L} \mathrm{h}^{-1}$ and a side channel of $12 \mu \mathrm{L} \mathrm{h}^{-1}$. As a result of this, a large part of the channel does not provide any functionality. In addition, the shear stresses on the cells will be proportionally higher for increased flow rates rendering them unsuitable for delicate mammalian cells.

\section{Sigmoid gradients}

Sigmoidal concentration gradients generated using microfluidics have been used in the literature for various biological assays including cell chemotaxis and drug testing. However, the significance of a sigmoidal profile is not fully established, therefore, testing several biological responses with sigmoidal concentration gradients is essential to understand different biological phenomena. In one example, sigmoid odor gradients were assayed for chemotactic studies of sensory experience of $C$. elegans by observing their movement in the gradients. ${ }^{28}$ The system reported here is extremely adaptable, so that change in flow rates can result in a notable difference capable of generating exponential or sigmoidal gradients. To generate a sigmoid concentration gradient, only one side channel was used to flow fluorescein dye solution along with the main channel to flow water, while the second side channel was left inactive. The ability of achieving a suitable gradient by means of simple modifications demonstrates a significant advantage of the reported design. Fig. 3a shows a sigmoidal concentration gradient of fluorescein generated using a side channel flow rate (SC) of $7 \mu \mathrm{L} \mathrm{h}^{-1}$ and a main channel flow rate (MC) of $10 \mu \mathrm{L} \mathrm{h}^{-1}$.
The experimental data pertaining to concentration was analyzed at two cross-sections, $X Y$ and $X^{\prime} Y^{\prime}$. These correspond to lines along the width of the main channel at a position closest to the side inlet port A and closest to the outlet of the device, respectively. As expected from a diffusion based phenomenon, mass of the solute should be conserved along the width and down the length of the main channel. Along the length of the channel, the concentration is expected to be more diffused and the rate of diffusion should be proportional to the slope of the concentration in small segments along the profile, Fig. 3b. Along the length of the channel and towards the dye edge, the concentration changes almost linearly at the rate of $5 \%$ per millimeter. The experimental data was fitted to a sigmoidal equation. A regression coefficient of 0.99 was obtained, which shows that gradient profiles generated under the previously specified parametric conditions accurately match a standard sigmoid. The fitted equation is given by the following logistic function:

$$
y=\frac{a-b}{1+\left(\frac{x}{x_{0}}\right)^{p}}+b
$$

where $x_{0}$, is the centre value of the data set, $p$ is the power, and $a$ and $b$ are the initial and final $y$ values respectively.

Fig. 3c contains comparative plots exhibiting variations in the concentration gradient profile for increasing $\mathrm{MC}$ flow rate by $4 \mu \mathrm{L} \mathrm{h}^{-1}$ steps, while keeping the SC flow rate constant at $7 \mu \mathrm{L} \mathrm{h}^{-1}$. Shorter fluorescein dye saturation regions are created for higher MC flow rates, which stems from the increased advection at higher MC flow rates resulting in more diffusant being carried away towards the outlet. Interestingly, gradients were shifted to the left or the right with respect to the center of the channel simply by modulating the MC flow rate and without significantly affecting the profiles. The gradient profile was repositioned to the left by approximately $200 \mu \mathrm{m}$ when the flow rates ratio (MC/SC) was doubled. It was also observed that, by lowering both of the MC and SC flow rates, the saturation regions can be shortened thereby increasing the linear region.
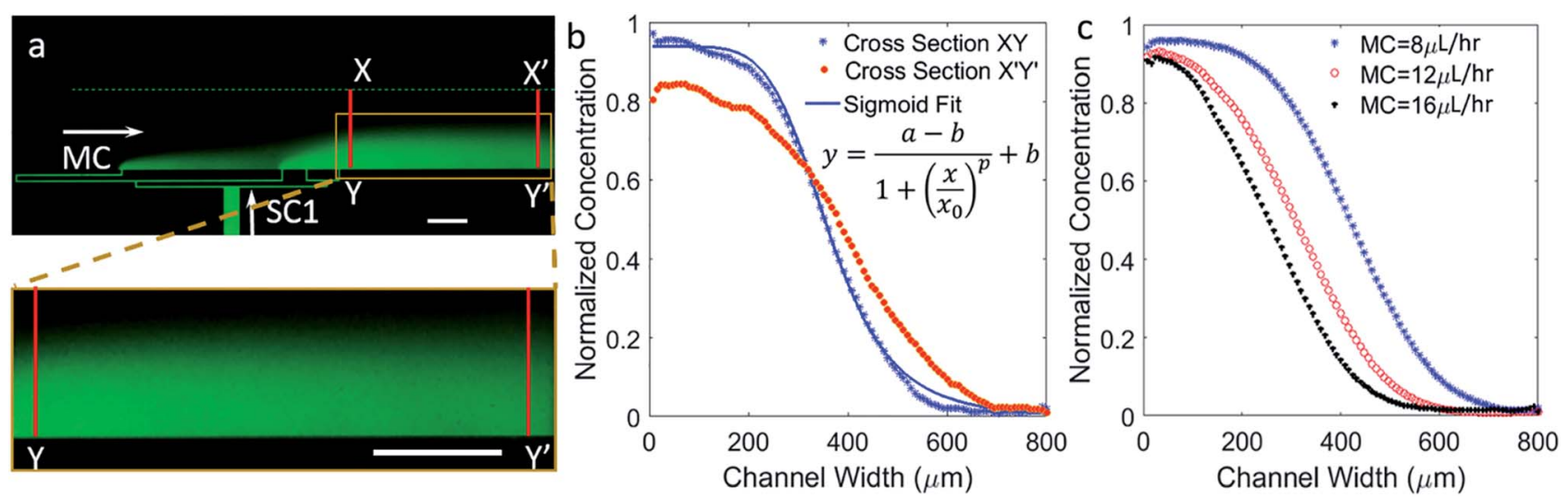

Fig. 3 Controllable concentration gradients with sigmodal profiles. (a) The device containing a fluorescein dye solution for sigmoidal gradient generation (scale bar is $400 \mu \mathrm{m}$ ). Enlarged image of the gradient is shown at the bottom (scale bar is $500 \mu \mathrm{m}$ ). (b) Normalized concentration profiles plotted along $X Y$ and $X^{\prime} Y^{\prime}$ lines shown in (a). The sigmoidal fitting data for the $X Y$ line profile is plotted as a solid line. (c) Effect of MC flow rate variation when the SC flow rate was kept constant at $7 \mu \mathrm{L} \mathrm{h}^{-1}$. The gradient profile can be repositioned within the main channel by only modulating the MC flow rate. 


\section{Parabolic gradients}

Concentration gradients with parabolic-, hill-, or bell-shaped profiles are commonly used to investigate chemotaxis ${ }^{29}$ and were established using microfluidics. ${ }^{\mathbf{1 3 0} 30}$ Other biological applications of bell-shaped gradients include determining the optimum concentration of viral infection on cell culture ${ }^{\mathbf{3 1}}$ and intracellular enzyme detection following cell lysis, ${ }^{32}$ to name a few. The use of parabolic gradients within a microfluidic setup is also of interest when studying chemotaxis under competing concentration gradients. ${ }^{33}$

To generate a parabolic profile with the developed microfluidic device, both side channels were pumped with the fluorescein dye solution, permitting the dye to diffuse into the center of the channel resulting in the lowest dye concentration in the middle. Fig. 4a shows an experimental image of fluorescein dye diffusing to form a parabolic profile when the SC and MC flow rates were $12 \mu \mathrm{L} \mathrm{h}^{-1}$ and $40 \mu \mathrm{L} \mathrm{h}^{-1}$, respectively. Fig. $4 \mathrm{~b}$ show the change in the gradient profile downstream the main channel. As fluids move downstream of the channel by means of convective mass flow, fluorescein molecules diffuses in the lateral direction. Therefore, flattening of the gradient profile is apparent with progressive increase in the dye concentration in the center and decrease in the two ends of the profile. In addition, the shape of the concentration profile obtained along $X Y$ is found to be more Gaussian in nature, but as the dye moves further down the channel, the profile along $X^{\prime} Y^{\prime}$
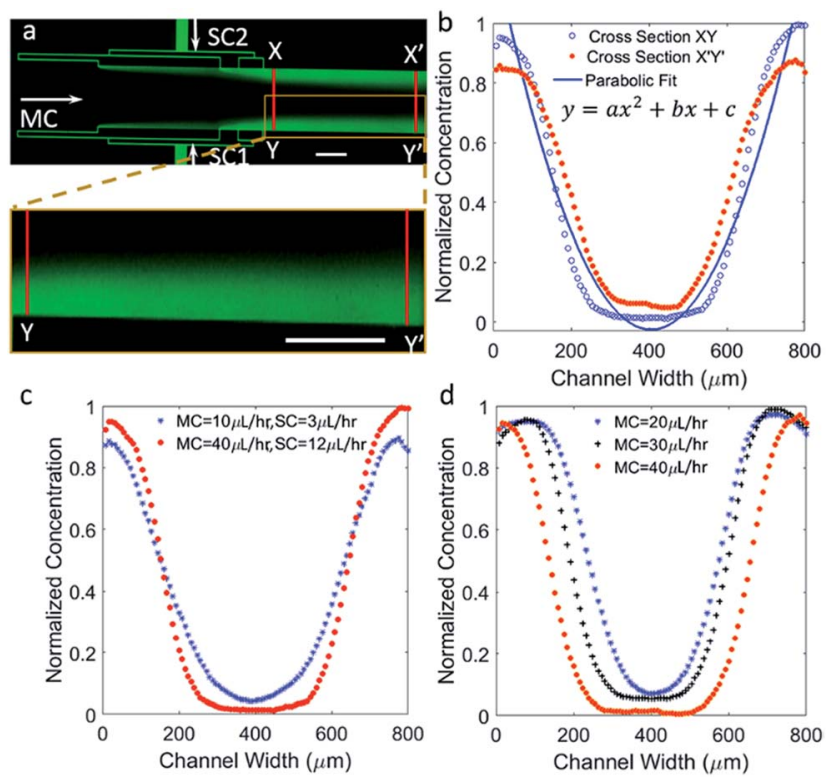

Fig. 4 Modulation of parabolic concentration gradients. (a) The device containing a fluorescein dye solution for parabolic gradient generation (scale bar is $400 \mu \mathrm{m}$ ). Enlarged image of the gradient is shown at the bottom (scale bar is $500 \mu \mathrm{m}$ ). (b) Normalized concentrations plotted along $X Y$ and $X^{\prime} Y^{\prime}$ lines in (a). The parabolic fitting data for the $X Y$ line profile is plotted as a solid line. (c) Parabolic profile variation when the MC and SC flow rates were decreased by a factor 4 . The shape can be modulated by changing the flow rates while keeping their ratio fixed. (d) Effect of the MC flow rate when the SC flow rate was kept constant at $10 \mu \mathrm{L} \mathrm{h}^{-1}$. turns parabolic. The experimental data was fitted to an equation for a parabola. A regression coefficient of 0.94 was obtained. The fitted equation is $y=a x^{2}+b x+c$, where $a, b$ are coefficients and $c$ is a constant.

In Fig. 4c, profiles were plotted along line $X Y$ to demonstrate the controllability of the profile shape by modulating the MC and SC flow rates. By reducing the flow rates, the profile becomes more parabolic, shown here for a reduction in flow rates by a factor of 4 . This is also accompanied by slope changes in the linear regions of the curve, which add functionality to modulate the profile on demand. The effects of changes in the MC flow rates, while keeping the SC flow rates constant at $10 \mu \mathrm{L} \mathrm{h}^{-1}$, are shown in Fig. 4d. This is also associated with moving both arms of the gradient by minimizing and lifting the central saturation part with minimal change with the slope. As mass transport is dominated by advection over diffusion, higher MC flow rates are expected to minimize the central saturation part, thus reposition the gradient arms further away from each other's. This is also of interest when introducing different chemokines through the side channels, where the distance between the opposing concentration gradients can be controlled by modulating the flow rates.

\section{Exponential gradients}

The study of exponential gradients is important as they are found to guide several in vivo processes. In particular, the guidance of neuronal growth cones is influenced by exponential gradients of chemotropic molecules during embryo development. ${ }^{34}$ Rosoff et al. has demonstrated rat dorsal root ganglion guidance using exponential gradients of Nerve growth factor, which was found to have interesting implications for axonal regeneration. ${ }^{35}$ In this work, the main parametric change necessary to generate an exponential concentration gradient from a sigmoidal profile is an increase in the ratio of MC and SC flow rates, which in turn causes suppression of the dye close to one wall of the main channel. For example, in Fig. $3 \mathrm{~b}$, the ratio of $\mathrm{MC} / \mathrm{SC}$ flow rates is 1.4 , whereas, in Fig. $5 \mathrm{~b}$, the ratio of $\mathrm{MC} /$ SC flow rates is 4 to generate an exponential gradient. Fig. $5 \mathrm{a}$ and b show surface concentration plots and line profiles corresponding to cross-sections $X Y$ and $X^{\prime} Y^{\prime}$. An exponential fitting function of $y=a \mathrm{e}^{b x}$ was used to plot a fitting curve, and a regression coefficient of 0.95 was obtained. In Fig. $5 \mathrm{c}$, the flow rates of both MC and SC were decreased by a factor of 6 , while keeping their ratio constant, in which causing modulation in the profile shape. As shown in Fig. 5 d, the exponential gradient was shifted to the left by around $100 \mu \mathrm{m}$ upon doubling the MC/ SC flow rates ratio, while observing minimal alterations to the slope. Similar to the sigmoidal and parabolic profiles, exponential gradients can be re-positioned within the channel by controlling the MC flow rate only. Moving concentration gradients are of interest for several applications, such as for challenging cells with moving gradients to study their dynamics during chemotaxis ${ }^{36,37}$ and for studying cell desensitization. ${ }^{38}$

Stable and well defined gradients with minimal fluid shear stresses are important for biological studies performed over extended time durations. The simple device configuration 

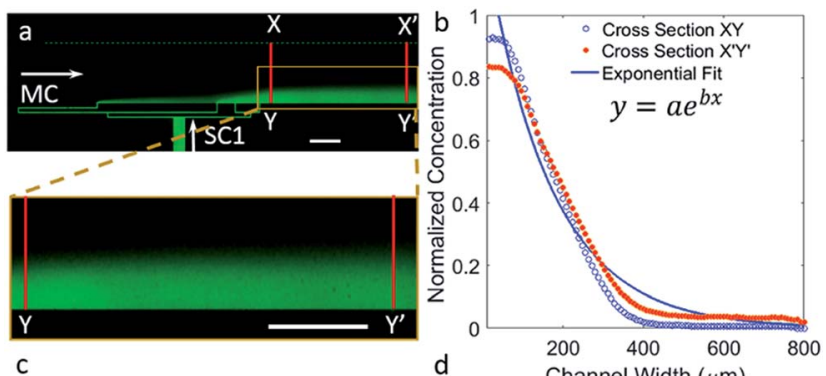

C

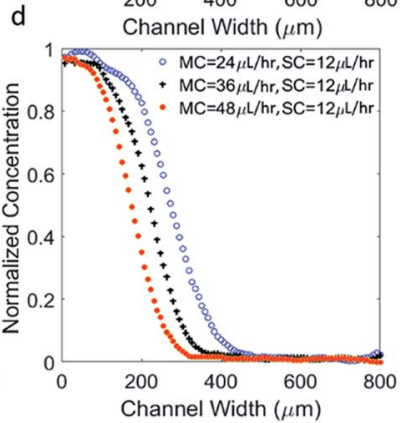

Fig. 5 Exponential concentration gradients. (a) A fluorescent micrograph showing the generated exponential concentration gradient (scale bar is $400 \mu \mathrm{m}$ ). Enlarged image of the gradient is shown at the bottom (scale bar is $500 \mu \mathrm{m}$ ). (b) Exponential profiles obtained at cross-sections $X Y$ and $X^{\prime} Y^{\prime}$. The blue solid line represents the fit to experimental data points $\left(y=a e^{b x}\right)$. Flow rates of $12 \mu \mathrm{L} \mathrm{h}^{-1}$ and $48 \mu \mathrm{L} \mathrm{h} \mathrm{h}^{-1}$ were used for SC and MC, respectively. (c) Exponential gradient profile variation when SC and MC flow rates were six folds higher. (d) Effect of the $M C$ flow rate variation when the SC flow rate was kept constant at $12 \mu \mathrm{L} \mathrm{h} \mathrm{h}^{-1}$

allowed us to generate and maintain clear and stable concentration gradients inside the main channel of the device. The concentration gradient simulations and experiments have been performed at $25{ }^{\circ} \mathrm{C}$. However, it is more desirable to maintain biological cells and the buffer at $37^{\circ} \mathrm{C}$. If the experiments reported here were performed in a temperature controlled chamber, the diffusion coefficient would vary according to the Stokes-Einstein relationship. For fluorescein the calculated increase in the diffusion coefficient was approximately $20 \%$. Testing was carried out by means of simulation, and it was determined that this change in diffusion coefficient did not result in a noticeable concentration profile variation, as shown in Fig. S3. $\dagger$

At the beginning of the experiments, tiny air bubbles were trapped in the channels, creating flow disturbances. Active fluid flow removed these bubbles in a couple of minutes, and nonpulsatile stable flow profiles were achieved. As mentioned previously, various gradient profiles were obtained just by altering MC and SC flow rates. The resulting stable concentration gradient shape and slope was achieved in under two minutes. The stability of generated profiles was determined from 5 minute time-lapse videos, and data point intensities were measured every 5 seconds. The mean and standard deviations were determined at 3 spatial points: the fluorescent dye saturation and diffusion regions, and the buffer saturation region. The coefficient of variation was determined over the entire 5 minute duration and was found to be $6.4 \%$, which provides good stability for biological studies.

\section{Conclusions}

The device presented here is capable of generating multiple concentration gradient profiles using four orthogonal channels connected to each side of the main channel. This simple device is easy to operate since overall channel lengths are shorter compared to other gradient generating devices in the literature. This reduces commonly encountered issues in microfluidic devices such as bubbles and clogging. In addition, the shorter length of the channels used here provides lowers hydrodynamic resistance compared to complex tree networks, thereby eliminating issues such as leakage between PDMS and the inlet fittings and long response time. In this device, switching between gradient profiles was achieved by only altering fluid flow rates without the need for any physical design modifications. The three gradient profiles characterized here by means of flow rate modifications are sigmoidal, parabolic, and exponential. These studies demonstrated the ability to alter gradient shape and position within the main channel by only modulating the flow rates. In this work, the two side channels are only active for the parabolic profile. If the flow rates of the channels are altered simultaneously in an unequal manner, the high flow rate fluid from the side channel will occupy a larger portion of the main channel, creating an unsymmetrical profile. Advection based chaotic mixing does not happen in this laminar flow system. However, advection is responsible for spatially regulating the rate of diffusion, thereby altering concentration profiles at various flow rates. One of the limitations of this device is the presence of saturation regions within the exponential and parabolic profiles. Simulation work indicated that by increasing the number of side channels beyond 4, fluid can be dispensed in smaller quantities through more number of side channels. This will decrease saturation regions both in the upper and lower concentration bounds. However, with increased number of side channels, the possibility of suppressed flow increases from certain side channels due to negative or zero pressure. Hence, in order to fully utilize a device with more number of side channels, finite element or analytical optimization studies need to be performed to eliminate such drawbacks.

\section{Conflicts of interest}

There are no conflicts to declare.

\section{Acknowledgements}

We acknowledge funding from the ADEC Award for Research Excellence (A2RE) 2015, Abu Dhabi Education Council, Abu Dhabi, UAE, and Seed Grant (AJF201523) from Al Jalila Foundation, Dubai, UAE. The authors thank Dr Thomas Gervais for the scientific discussions. The authors thank Dr Muhammedin Deliorman and Dr Mohammed Daqaq for their critical readings of the manuscript.

\section{References}

1 S. J. Allen, S. E. Crown and T. M. Handel, Annu. Rev. Immunol., 2007, 25, 787-820. 
2 P. Devreotes and C. Janetopoulos, J. Biol. Chem., 2003, 278, 20445-20448.

3 C. S. Blengini, M. E. Teves, D. R. Unates, H. A. Guidobaldi, L. V. Gatica and L. C. Giojalas, Asian J. Androl., 2011, 13, 769-773.

4 W. A. Muller, Vet. Pathol., 2013, 50, 7-22.

5 J. A. Madri and D. Graesser, Dev. Immunol., 2000, 7, 103-116.

6 L. Schneider, M. Cammer, J. Lehman, S. K. Nielsen,

C. F. Guerra, I. R. Veland, C. Stock, E. K. Hoffmann, B. K. Yoder, A. Schwab, P. Satir and S. T. Christensen, Cell. Physiol. Biochem., 2010, 25, 279-292.

7 E. T. Roussos, J. S. Condeelis and A. Patsialou, Nat. Rev. Cancer, 2011, 11, 573-587.

8 G. H. Wadhams and J. P. Armitage, Nat. Rev. Mol. Cell Biol., 2004, 5, 1024-1037.

9 T. Ahmed, T. S. Shimizu and R. Stocker, Integr. Biol., 2010, 2, 604-629.

10 H. Mao, P. S. Cremer and M. D. Manson, Proc. Natl. Acad. Sci. U. S. A., 2003, 100, 5449-5454.

11 A. J. Muinonen-Martin, D. M. Veltman, G. Kalna and R. H. Insall, PLoS One, 2010, 5, e15309.

12 N. Kramer, A. Walzl, C. Unger, M. Rosner, G. Krupitza, M. Hengstschlager and H. Dolznig, Mutat. Res., 2013, 752, 10-24.

13 J. Diao, L. Young, S. Kim, E. A. Fogarty, S. M. Heilman, P. Zhou, M. L. Shuler, M. Wu and M. P. DeLisa, Lab Chip, 2006, 6, 381-388.

14 S. K. W. Dertinger, D. T. Chiu, N. L. Jeon and G. M. Whitesides, Anal. Chem., 2001, 73, 1240-1246.

15 N. L. Jeon, S. K. W. Dertinger, D. T. Chiu, I. S. Choi, A. D. Stroock and G. M. Whitesides, Langmuir, 2000, 16, 8311-8316.

16 C. G. Yang, Y. F. Wu, Z. R. Xu and J. H. Wang, Lab Chip, 2011, 11, 3305-3312.

17 M. A. Qasaimeh, T. Gervais and D. Juncker, Nat. Commun., 2011, 2, 464.

18 M. A. Qasaimeh, S. G. Ricoult and D. Juncker, Lab Chip, 2013, 13, 40-50.

19 A. T. Brimmo and M. A. Qasaimeh, IEEE Nanotechnology Magazine, 2017, 11, 20-31.

20 C. Guermonprez, S. Michelin and C. N. Baroud, Biomicrofluidics, 2015, 9, 054119.
21 M. A. Qasaimeh, M. Astolfi, M. Pyzik, S. Vidal and D. Juncker, 40th Annual Northeast Bioengineering Conference (NEBEC), April 25-27 2014, pp. 1-2.

22 S. K. W. Dertinger, X. Jiang, Z. Li, V. N. Murthy and G. M. Whitesides, Proc. Natl. Acad. Sci. U. S. A., 2002, 99, 12542-12547.

23 T. F. W. Sloan, M. A. Qasaimeh, D. Juncker, P. T. Yam and F. Charron, PLoS Biol., 2015, 13, e1002119.

24 D. van Noort, S. M. Ong, C. Zhang, S. Zhang, T. Arooz and H. Yu, Biotechnol. Prog., 2009, 25, 52-60.

25 R. Stocker, J. R. Seymour, A. Samadani, D. E. Hunt and M. F. Polz, Proc. Natl. Acad. Sci. U. S. A., 2008, 105, 42094214.

26 S. Geremia, M. Campagnolo, N. Demitri and L. N. Johnson, Structure, 2006, 14, 393-400.

27 S. Varma and J. Voldman, Lab Chip, 2015, 15, 1563-1573.

28 J. Larsch, S. W. Flavell, Q. Liu, A. Gordus, D. R. Albrecht and C. I. Bargmann, Cell Rep., 2015, 12, 1748-1760.

29 N. Li Jeon, H. Baskaran, S. K. Dertinger, G. M. Whitesides, L. Van de Water and M. Toner, Nat. Biotechnol., 2002, 20, 826-830.

30 Y. Zhou, Y. Wang, T. Mukherjee and Q. Lin, Lab Chip, 2009, 9, 1439-1448.

31 G. M. Walker, M. S. Ozers and D. J. Beebe, Sens. Actuators, B, 2004, 98, 347-355.

32 E. A. Schilling, A. E. Kamholz and P. Yager, Anal. Chem., 2002, 74, 1798-1804.

33 B. $\mathrm{Hu}$ and Y. Tu, Biophys. J., 2013, 105, 276-285.

34 C. M. Isbister, P. J. Mackenzie, K. C. W. To and T. P. Connor, J. Neurosci., 2003, 23, 193.

35 W. J. Rosoff, J. S. Urbach, M. A. Esrick, R. G. McAllister, L. J. Richards and G. J. Goodhill, Nat. Neurosci., 2004, 7, 678-682.

36 A. J. Aranyosi, E. A. Wong and D. Irimia, Lab Chip, 2015, 15, 549-556.

37 M. A. Qasaimeh, M. Astolfi, M. Pyzik, S. Vidal and D. Juncker, The seventeenth International Conference on Miniaturized Systems for Chemistry and Life Sciences, Freiburg, Germany, October 27-31 2013, pp. 2007-2009.

38 T. M. Keenan, C. W. Frevert, A. Wu, V. Wong and A. Folch, Lab Chip, 2010, 10, 116-122. 